\title{
CANCER CELL SEGMENTATION AND DETECTION USING NC RATIO
}

\author{
ANALYSIS \\ S. Manjubharathi ${ }^{1}$, S. Saraswathi \\ ${ }^{1}$ M.E-Communication Systems, Department of Electronics and communication, Mahendra Engineering College \\ ${ }^{2}$ Assistant professor, Department of Electronics and communication, Mahendra Engineering College
}

\begin{abstract}
This paper presents a new progressive segmentation for skin cancer cells of third harmonic microscopic image. This method is based on detection of early diagnosis of skin cancer cells. The experimental result shows that the proposed algorithm gives Competitive results compared to the previous works deals with watershed transform.
\end{abstract}

Keywords- Cell segmentation, Computer tomography, Graph-cut algorithm, Nuclear to Cytoplasm (NC) ratio, Red Blood Cells, Third harmonic microscopy, White blood cells.

\section{INTRODUCTION}

The early diagnosis of the skin cancer is detected by the physical biopsy procedure it involves the removal of the affected tissue from a living subject, and by using microscope it was evaluated [1]. This may lead to spread of the cancer cell to the healthy cells and also it is more painful to the patients also. Skin cancer is an abnormal tissue seen in the lower epidermal layers of the skin. This will affect the skin layer from the usual function. So the physical structure of the skin layer also changes. The matured stage of these abnormal tissue results in damage of the overall layer of the lower epidermis. The image modalities are the computed tomography (CT), magnetic resonance image; ultrasounds imaging these are the best examples of well developed technologies of visualizing the internal structures of in vivo. Its capable of only visualize smaller than $10-1 \mathrm{~m}$. These methodologies compare the current manual counting and method from the art and state.
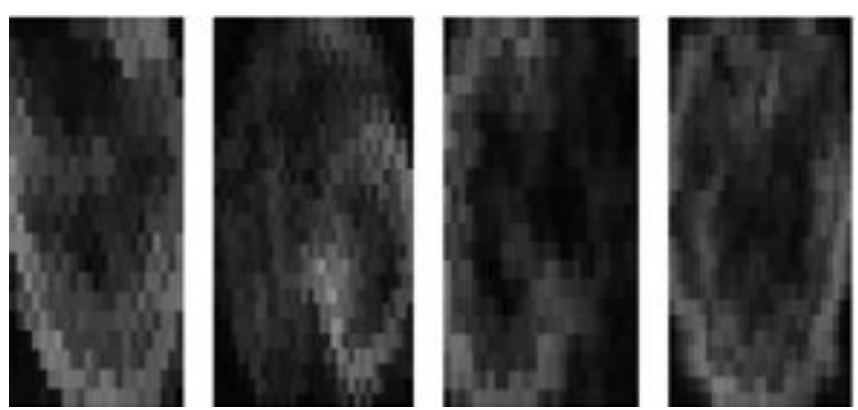

(a) $1=1.06 \quad$ (b) $l=5.92$ (c) $l=3.83$ (d) $1=2.158$
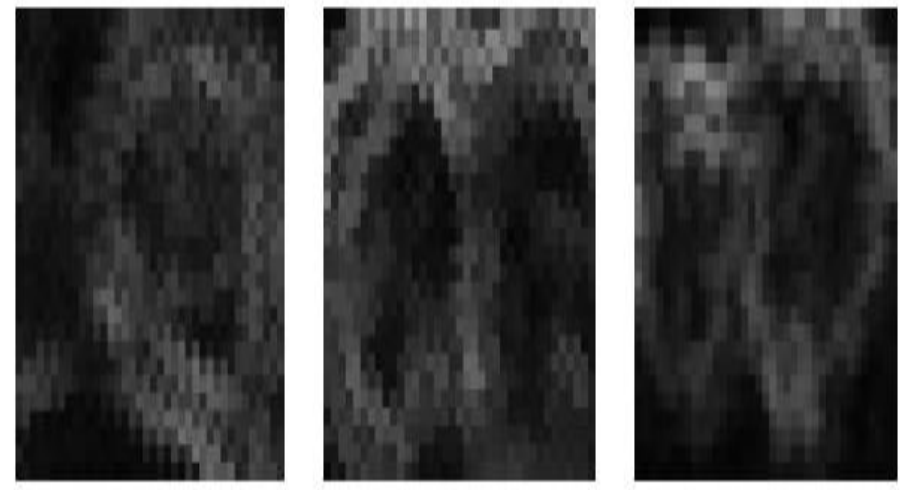

(e) $1=-1.45$

(f) $1=-15.16$

(g) $1=-5.60$

Fig 1.1: Cell detection by manual evaluation

It first represent the counting manually, and that results of the whole data and these process finally completed by the suggestion of how compactness of the calculation used in these regard.

\section{THIRD HARMONIC MICROSCOPY}

The third harmonic microscopy which is $1230 \mathrm{~nm}$ base which is useful identification of the noninvasive in vivo virtual biopsy on the skin, it has the penetration depth of $>300 \mu \mathrm{m}$ [3]. It produces the high resolution and penetrability at the same time. It produces much more penetrability in different types of skin also. The tumors originate in the basal layer of epidermis the pigment producing melanocytes. These melanomas are similar to moles; but there is some development in the moles. Most of the melanomas are brown and black, skin-colored, pink, red, purple, blue or white. It is 
mainly caused by UV EXPOSURE, intense, (frequently leading to sunburn). The good indicator to identify the early symptoms of the skin cancer is the nuclear to cytoplasm ratio [2]. The ratio of nuclear and the cytoplasm are constant for a certain cell type and it is increased in malignant neoplasm. This ratio is also used to characterize cells.

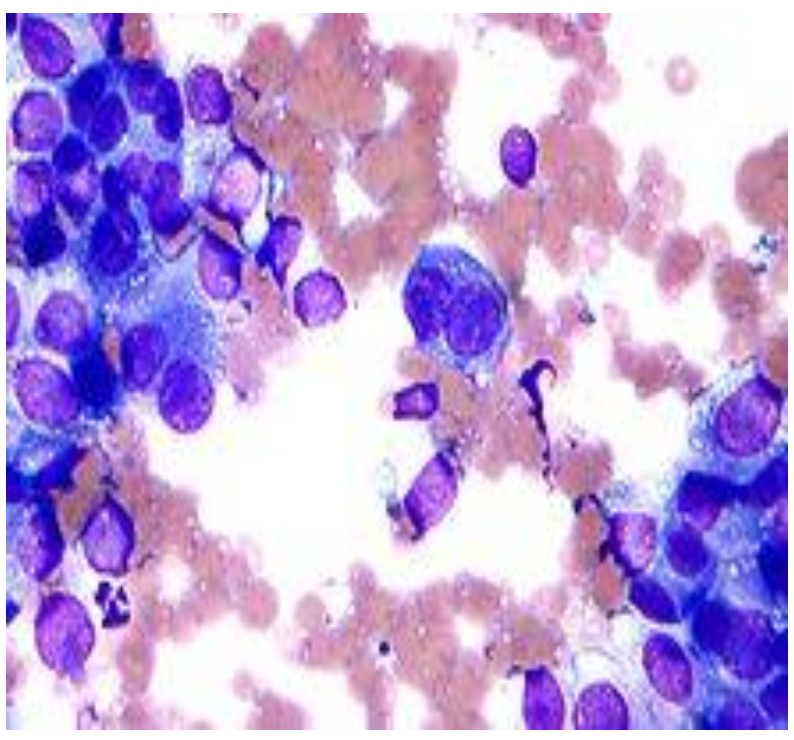

Fig 2.1: Micrograph of Melanoma

The cancer cells are not matured as the normal cells it larger than the normal cells. NC ratio can be used to diagnose cancers in some of the tissue and it is useful for clinical applications also.

This ratio also indicates the maturity of a cell, because if the cell matures the size of its nucleus generally decreases. The previous algorithms used for the cell segmentation are Region splitting and merging, region growing, image thresholding, snakes [5], [6] and balloon algorithms, watershed algorithm [5]. The image thresholding [4] it is the simplest method which provides good result in the high contrast image between the object and the background. Region splitting and merging and region growing produce over- segmentation problem and it does not have a constant segmentation result.

\section{IMPLEMENTATION}

The main objective of graph cut method is completely automatic high level grouping of image pixels it divides an image into blobs and clusters [7]. A graph-cut method is defined as

$$
\mathrm{Gc}=(\mathrm{n}, \mathrm{Nc})
$$

Where,

$\mathrm{n}$ is the nodes or vertices

$\mathrm{Nc}$ set of edges
It uses implicit representation of object boundaries. It is the only method which offers optimal object extraction for $\mathrm{N}$ dimensional images. The image segmentation is the most familiar and important method of IFT, it covers the both representation such as the boundary and then the region-based. Methods used for these algorithms are nuclei segmentation and cytoplasm segmentation. To measure the compactness [8] of the valid nuclei,

Nuclei validation $=\mathrm{A} / \mathrm{P}^{2}$

Here A is the total boundary of the object and P represents the perimeter of the object. Cytoplasm is validated by measuring the boundaries of the cytoplasm and nuclei it is represented as the boundary between the cytoplasm and inner boundary of the nuclei [9].

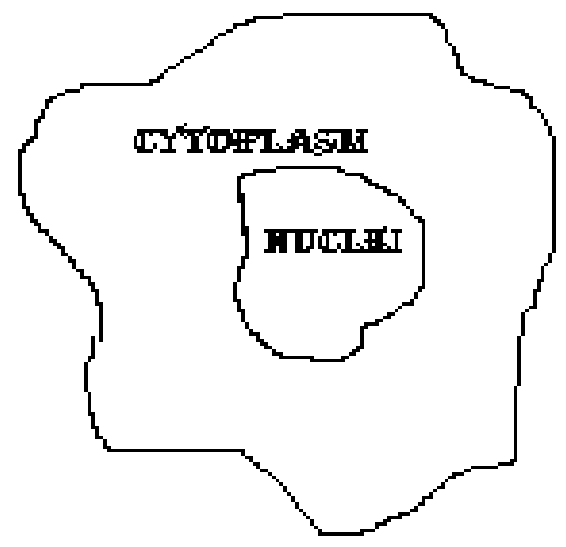

Fig 3.1: Cell structure

In graph-cut nodes indicates image pixels or voxels [6]. It is the best in optimal segmentation these method uses the S-T terminals it represent object and background. S-t subset of edges is represented as Cc€. It is separately noted as $\mathrm{G}(\mathrm{c})=(\mathrm{v}, € / \mathrm{c})$, Boundary and region are represented as

$$
\mathrm{e}(\mathrm{s})=\mathrm{r}\{\mathrm{p}\}+\mathrm{b}\{\mathrm{p}\}
$$

Where

$r(p)=\{€ R p(G c)$, Region representation

$\mathrm{b}(\mathrm{p})=€ \mathrm{~B}$ a,b

It uses dig out bare minimum cut and ceiling flow algorithms.

\subsection{Nuclei Segmentation}

In the nucleus segmentation, the design of the image was process are based on the connectivity. It is performed by the methods blob detection [9], nuclei initialization. The nuclei initialization is used to know the cell boundary which was followed by the outlier removal, distance transform. The 
process function as it first a binary image is created by the thresholding and then creating simplified images then the small residues are discarding.
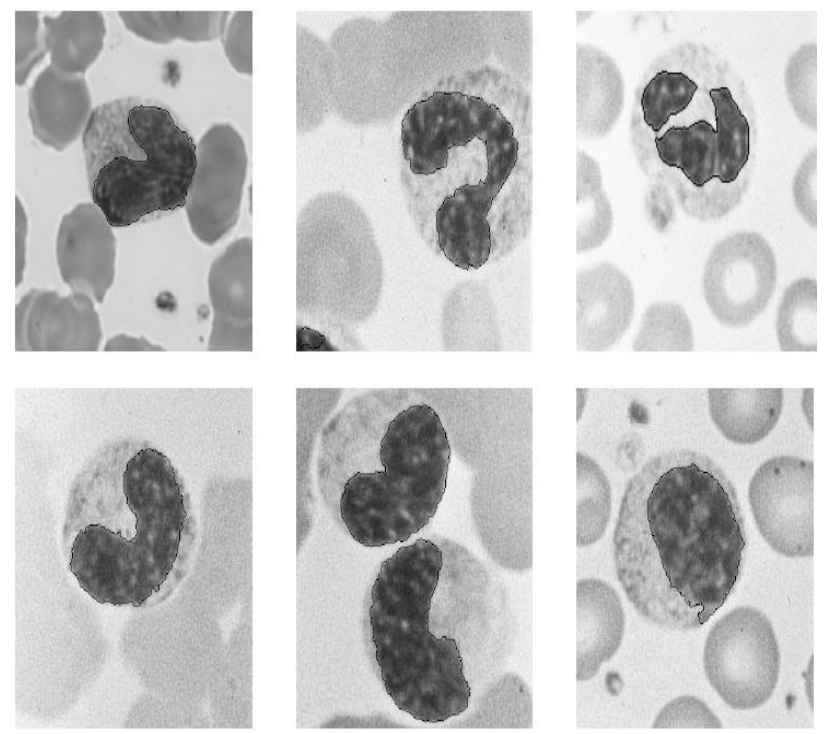

Fig 3.2: Nuclei segmentation

The WBC nucleus is used for the classification for counting differential process that is which based on the path-cost function. The boundaries are marked on internal and external map marking prospective of the consequent nuclei.

\subsection{Cytoplasm Segmentation}

The main process is to extract the cytoplasm from background and RBC. Morphological tool which capture their cell size. To obtain these function a morphological open using element increasing of the size. These opening between the element size and number of removed image object [10]. For this segmentation an convergence index filter is most apt for the low-contrast and noisy microscopy image.

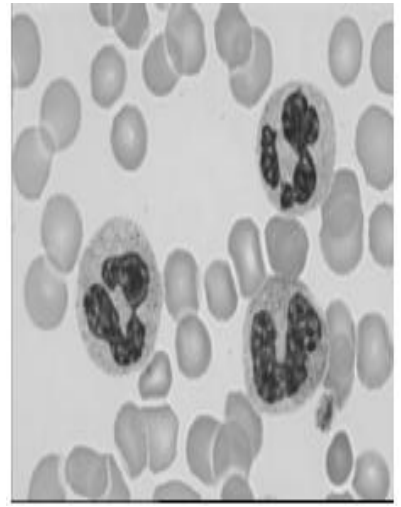

( a) original

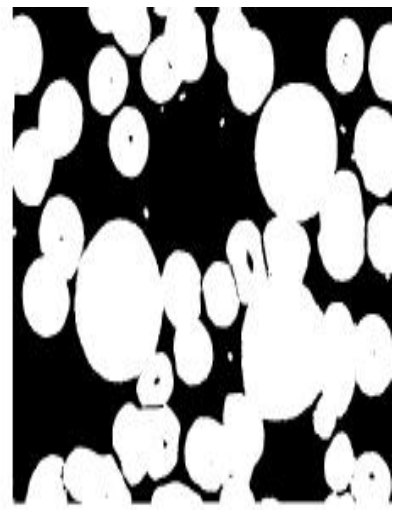

(b) threshold

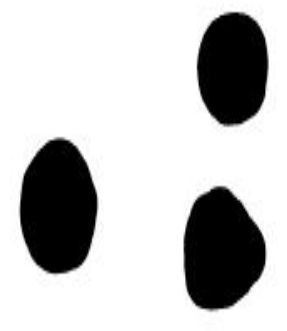

(c) opening

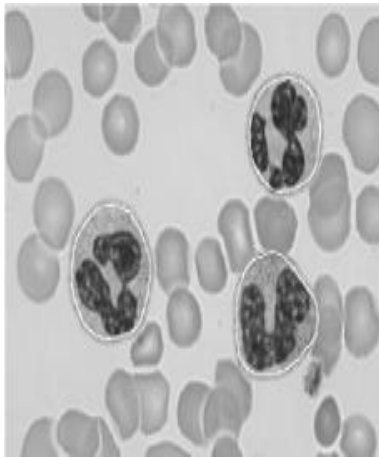

(d) segmentation
Fig 3.3: Cytoplasm segmentation format

The table I shows the cell segmented profile of the nuclei and then the cytoplasm cell area which measures the ratio between them. This is the best indicator of the cancer cells. In which the normal NC ratio is different from the cancer affected area. The cell was segmented automatically. The process is performed by the proposed algorithm.

The evaluated ratios and then their size are used in the clinical process after the discussion with the dermatologist and Radiologist. Table II it is the comparison of our proposed work with the previous work measures the mean and standard deviation it indicates the two set of ratio which was the evaluated $\mathrm{NC}$ ratios based on the catalog of THG images. The main dissimilarity in the future work is development and analyzes the biomedical images routinely with the manual assortment of cells for the cell segmentation of followed by $\mathrm{NC}$ ratio analysis.

These evaluated ratios are based on the cram of epidermis for some of the emblematic skin cancers resembling squamous cell carcinoma and actinic keratosis; these are finding out by the healthy cells. In the biomedical images the blare are used to deal with the automatic exposure of the prospective sources of the NC ratios as in table II. 
Table 1: Cell Segmentation Outline

\begin{tabular}{|l|l|l|l|}
\hline Cell index & Nuclei area & Cytoplasm area & Ratio \\
\hline 1 & 48 & 233 & 1.1957 \\
\hline 2 & 38 & 275 & 1.1300 \\
\hline 3 & 26 & 90 & 1.2609 \\
\hline 4 & 29 & 79 & 1.3333 \\
\hline 5 & 25 & 97 & 1.2323 \\
\hline 6 & 26 & 335 & 1.0712 \\
\hline 7 & 33 & 171 & 1.1792 \\
\hline 8 & 41 & 116 & 1.3305 \\
\hline 9 & 26 & 115 & 1.2051 \\
\hline 10 & 28 & 112 & 1.2281 \\
\hline Total & 320 & 1623 & - \\
\hline
\end{tabular}

Table 2: Comparison with Previous Method

\begin{tabular}{|l|l|l|}
\hline Measure & $\begin{array}{l}\text { Proposed } \\
\text { method }\end{array}$ & Exist method \\
\hline Mean & 1.2166 & 0.3100 \\
\hline $\begin{array}{l}\text { Standard } \\
\text { deviation }\end{array}$ & 0.0813 & 0.0140 \\
\hline $\begin{array}{l}\text { Time take to } \\
\text { process }\end{array}$ & $\begin{array}{l}10-5 \text { seconds per } \\
\text { image }\end{array}$ & $\begin{array}{l}\text { Several } \\
\text { minutes pre } \\
\text { image }\end{array}$ \\
\hline
\end{tabular}

\section{RESULTS AND DISCUSSION}

By using the proposed method a 15bit of the binary images are $512 * 512$ pixel image took $10-5$ seconds. It also usable in the photo and video editing. The key aspiration is to offer the concept of object mining. In the field of medical imaging we acquire a 3D medicinal dimension. The corrections in these are performed with in a several second. These proposed algorithm increases the pace of scrutiny of medical images it not only provide scrutiny of images it also afford the segmentation results with a unvarying accuracy. In the manual assortment the segmentation outcome takes quite a few minutes and also it provides extremely deprived result.

The proposed exertion hoards time and provides a convincing result with the construal and discussion by the medical doctors. Therefore the method has significant potential for biomedical imaging analysis

\section{CONCLUSIONS}

By using the $\mathrm{NC}$ ratio the skin cancer is analyzed and the early diagnoses of the diseases are identified. In this paper, a computer aided design for cancer cell and the NC ratio analysis in automatic cell segmentation was proposed.
The experimental result shows that the anticipated method provides objective segmentation results with the soaring efficiency and steady accuracy. It performed for the analysis of the biomedical imaging and it also satisfies the clinical requirement in many other applications. This is stouter than the manual advance; hence these can be used instead of the individual judgments of analyzer and the exhaustion of the medical people. Our method is used for the global cosmetics companies that evaluate and scrutinize how a certain manufactured goods may impinge on the cells of perspective users.

\section{REFERENCES}

[1]. Sun C.-K (2012)"automatic cell segmentation using the $\mathrm{NC}$ ratio analysis of third harmonic microscopic medical image".

[2]. Sun C.-K., (2005)“"Higher harmonic generation Microscopy," Adv. Brioche. Eng. /Biotechnology, vol. 95, pp. 17-56.

[3]. Chen S .-Y., Chen S.-U. H.-Y. Wu,Lee W.-J. Liao Y.-H., and Sun C.-K., "In vivo Virtual biopsy of human skin by using Noninvasive higher harmony generation Microscopy, (2010.)" IEEE J. Sel. Topics Quantum Electron, vol. 16, no. 3, pp. 478-492,

[4]. Gonzalez R. C. And Woods R. E.,(2002)" Digital Image Processing”. Englewood Cliffs, NJ, USA: Prentice-Hall.

[5]. Meyer. F, (1994) "Topographic distance and watershed lines,” Signal Process., vol. 38, pp. 113-125.

[6] Wang Y.-Y., Sun C.-C., Lin. K and M.-S. Ju, (2006)"Nerve cell Segmentation via multi-scale gradient watershed hierarchies," in Proc.28thAnnul. Int. Conf. IEEE Engineering in Medicine and Biology Soc., pp. 4310-4313.

[7]. Yuri Boykovgareth, Funka-Lea,(2006),"graph cut and n-d image"vol.70 109-131.

[8]. MacEachren A. M., (1985) "Compactness of geographic shape: ComparisonAnd evaluation of measures," Geografiska Annaler. Human Geography, ser. B, pp. 53-67.

[9]. Geraud.T, Mangin J. F., Bloch. I, and Maitre.H,(1995) "Segmenting the Internal structures in 3D MR images of the brain by Markova Relaxation on a watershed based adjacency graph," in Proc. IEEE Int.Conf. Image Processing, Washington, DC, vol. 3, pp. 548-551.

[10]. Quelhas. P, Marcuzzo. M,. Mendonca.A. M, and A. Campilho, (2010)"Cell Nuclei and cytoplasm joint segmentation using the sliding band filter," IEEE Trans. Med. Image., vol. 29, no. 8, pp. 1463-1473. 